\title{
Genotyping and Drug Resistance Profile of Clinical Isolates of Candida albicans from Vulvovaginal Candidiasis in the Eastern China
}

\author{
Nan Hong • Yan Lei $\cdot$ Huan Chen $\cdot$ Xiaofei Chen $\cdot$ Kin Ming Tsui $\cdot$ \\ Danyang Hu • Wanqing Liao $\cdot$ Liang Yan $\cdot$ Hong Zhang $\cdot$ Rongfen Zhao • \\ Gang Wu $\cdot$ Nong Yu $\cdot$ Shuwen Deng
}

Received: 9 October 2021 / Accepted: 1 January 2022/Published online: 24 January 2022

(C) The Author(s) 2022

\begin{abstract}
A total of 244 Candida albicans isolates recovered from vulvovaginal candidiasis (VVC) patients in Suzhou, Eastern China, were investigated. According to CLSI documents M27-A4 and M59-3ed/ M60-2ed, the MIC geometric means of nine antifungals in increasing order were micafungin $(0.048 \mathrm{mg} /$ $\mathrm{L})$ anidulafungin $(0.132 \mathrm{mg} / \mathrm{L}), \quad$ caspofungin $(0.19 \mathrm{mg} / \mathrm{L})$, itraconazole $(0.23 \mathrm{mg} / \mathrm{L})$, posaconazole $(0.25 \mathrm{mg} / \mathrm{L})$, voriconazole $(0.28 \mathrm{mg} / \mathrm{L}), 5$-flucytosine $(0.44 \mathrm{mg} / \mathrm{L})$, amphotericin B $(0.49 \mathrm{mg} / \mathrm{L})$ and
\end{abstract}

Handling Editor: J.-P. Bouchara.

Supplementary Information The online version contains supplementary material available at https://doi.org/10.1007/ s11046-022-00616-x.

X. Chen $\cdot$ D. Hu $\cdot$ H. Zhang $\cdot$ R. Zhao .

N. Yu $\cdot$ S. Deng $(\bowtie)$

Department of Medical Microbiology, The People's

Hospital of Suzhou New District, Suzhou, Jiangsu, China

e-mail: shuwen.deng@gmail.com

N. Hong

Department of Dermatology, South China Hospital,

Health Science Center, Shenzhen University, Shenzhen,

China

N. Hong $\cdot$ H. Chen

Department of Dermatology, Jinling Hospital, Nanjing

University School of Medicine, Nanjing, China fluconazole $(2.01 \mathrm{mg} / \mathrm{L})$ respectively. Of note, $6.5 \%$ (16/244) C. albicans isolates showed resistance mainly to anidulafungin (mono-echinocandin resistance), while voriconazole had the lowest susceptibility rate of $34.8 \%(85 / 244)$, followed by fluconazole $59.4 \%$ (145/244), respectively. All isolates were genotyped by allelic combination of 3 microsatellite markers (CEF3, CAIII and LOC4). A total of 129 different allelic genotypes were identified, in which seven different clades were recognized with a discriminatory power of 0.96 . Genotypes A-D were present in $35 \%$ of the isolates. In conclusion, decrease in antifungal drug susceptibility to $C$. albicans isolates from VVC is alarming. Our findings revealed the genetic diversity of $C$. albicans isolates among VVC

Y. Lei

Department of Dermatology, Peking Union Medical

College Hospital, Chinese Academy of Medical Sciences and Peking Union Medical College, Beijing, China

K. M. Tsui

Division of Infectious Diseases, Faculty of Medicine, University of British Columbia, Vancouver, Canada

K. M. Tsui

Department of Pathology, Sidra Medicine, Doha, Qatar

W. Liao

Shanghai Key Laboratory of Medical Molecular Mycology, Department of Dermatology, Changzheng Hospital, Second Military Medical University, Shanghai, China 
patients and provided insights into the molecular epidemiology of Candida infections in China.

Keywords Genetic diversity · Antifungal susceptibility · Resistance - Candida albicans . Vulvovaginal candidiasis · China

\section{Introduction}

Vulvovaginal candidiasis (VVC) is one of the most common vaginitis caused by Candida albicans, which accounts for $80-95 \%$ of all episodes of VVC worldwide, though the number of non-albicans species (such as Candida glabrata) as etiological agents of $\mathrm{VVC}$ is increasing [1, 2]. An increasing prevalence of fungal resistance is also reported in antifungal surveillance studies globally [3-5]. Antifungal susceptibility testing of $C$. albicans isolates therefore plays a crucial role for appropriate and effective management strategies of VVC [5]. In addition, it is important to look into the population genetic structure and epidemiology of C. albicans infections because of increasing reports indicating dynamic changes in the antifungal susceptibility profile with different genotypes and geographic origins [6]. Thus, understanding the genetic diversity of $C$. albicans could help clinicians to implement appropriate diagnostic, therapeutic and preventive strategies [7, 8]. Microsatellite analysis is highly reproducible with strong discriminatory power and is widely applied in molecular typing of fungal pathogens [9]. In previous studies, microsatellite analysis has proved to be a powerful tool in investigating the relationship between genetic diversity and antifungal susceptibility of $C$. albicans isolates $[10,11]$. However, knowledge on the population genetic structure and antifungal susceptibility profile of isolates from VVC patients in China still remains limited. Therefore, in this study, we investigated the antifungal susceptibility profile on hundreds of $C$. albicans isolates from VVC patients in eastern China

\section{Yan}

Department of Dermatology, PLA General Hospital of Central Theater Command, Wuhan, China

G. Wu

Nanjing Chia Tai Tianqing Pharmaceutical Co., Ltd, Nanjing, China
(Suzhou area) based on M27-A4 and M59/M60 documents approved in 2020 for interpretive breakpoints and epidemiological cutoff (ECV). Also, we performed molecular typing utilizing three microsatellite loci (CAIII, CEF3, and LOC4) to investigate the genetic variability.

\section{Materials and Methods}

\section{Isolates and Identification}

A total of 244 vaginal C. albicans isolates were recovered from patients with vulvovaginal candidiasis in the People's hospital of Suzhou New District during Jan to Dec. 2018. The patients and case definition, vaginal samples collecting information have been reported in previous publication [5]. All isolates were identified to the species level by sequencing D1/D2 domain of $26 \mathrm{~S}$ ribosomal DNA gene as described previously [5]. Isolates information and GenBank accession numbers of D1/D2 sequences are listed in supplementary table 1 .

\section{Microsatellite Analysis}

Microsatellite genotyping was performed with all 244 C. albicans isolates, based on a panel of three different short-nucleotide repeat fragments, using fluorescently labeled primers CAIII (5'-Tamra -TTGGAATCACTTCACCAGGA-3', 5' ${ }^{\prime}$-TTTCCGTGGCATCAGTATCA-3'); CEF3 (5'- Hex-TTTCCTCTTCCT TTCATATAGAA- $3^{\prime}, 5^{\prime}$ - GGATTCACTAGCAGCAGACA-3'); LOC4 (5'- FAM -GTAATGATTACGGCAATGAC-3', 5' -AGAACGACGTGTACTATTGG$3^{\prime}$ ) [11]. A multiplex polymerase chain reaction (PCR) was performed in $10 \mu \mathrm{l}$ reaction volumes containing $5 \mu \mathrm{l}$ of Qiagen Multiplex PCR (2x, Lot 148031955), $0.25 \mu \mathrm{l}$ of each primer (forward and reverse), $3 \mu \mathrm{l}$ of $\mathrm{ddH}_{2} \mathrm{O}$, and $1 \mu \mathrm{l}$ of genomic DNA. PCR amplifications were performed in a thermocycler (BOECO, TCPro, Germany) operating with a temperature-cycling program that consisted of an initial denaturing step at $95^{\circ} \mathrm{C}$ for $15 \mathrm{~min}$, followed by 35 cycles of $30 \mathrm{~s}$ at $94{ }^{\circ} \mathrm{C}, 90 \mathrm{~s}$ at $57^{\circ} \mathrm{C}$, and $60 \mathrm{~s}$ at $72{ }^{\circ} \mathrm{C}$, with a final extension step of $10 \mathrm{~min}$ at $72{ }^{\circ} \mathrm{C}$. The size of the fragments was determined by addition of the GeneScan LIZ500 marker and subsequent analysis on the Applied Biosystems 3730 DNA analyzer. Assignment 
of repeat numbers in each marker was determined from the GeneScan data by using the Peak GeneMapper 5.0 software (Applied Biosystems, Foster City, CA, USA). Allele-sharing distance matrices were generated from the tandem repeat numbers and were used as input for UPGMA clustering analysis. The UPGMA clustering of the 244 C. albicans isolates was performed using $\mathrm{R}$ package phangorn. The UPGMA tree was then plotted using $\mathrm{R}$ (version 3.4.4). The discrimination power (DP) of the microsatellite genotyping method used in this study was calculated by the online calculator created by the university of the basque country (http://insilico.ehu.es/mini_tools/ discriminatory_power) which evaluates the probability of any pair of isolates to belong to distinct genotypes.

\section{Antifungal Susceptibility Testing}

All isolates were tested for in vitro susceptibility to nine antifungal drugs agents according to the CLSI reference guideline M27-A4 [12]. Antifungal drugs tested were anidulafungin (ANF), caspofungin (CAS), micafungin (MFG), amphotericin B (AmB), 5-flucytosine (5-FC), fluconazole (FLC), itraconazole (ITR), voriconazole (VRC) and posaconazole (POS). Anidulafungin and voriconazole were purchased from Toronto Research Chemicals Inc (Canada), micafungin was provided by Astellas Pharma (Japan), and remaining antifungals were obtained from SigmaAldrich. Candida parapsilosis ATCC 22019 and Candida krusei ATCC 6258 were used as control strains in all experiments. All isolates were subcultured onto Sabouraud Dextrose Agar at $35{ }^{\circ} \mathrm{C}$ for $24 \mathrm{~h}$ for viability and purity. Colonies were suspended in sterile saline, and the final inoculum concentration of the suspension was adjusted to $0.5-2.5 \times 10^{3} \mathrm{CFU} /$ $\mathrm{mL}$ with RPMI1640 broth medium. The 96 -well plates were incubated for 24 or $48 \mathrm{~h}$ at $35^{\circ} \mathrm{C}$, and the minimum inhibitory concentrations (MIC) were determined visually. Drug concentration ranges, time of MIC readings and interpretive breakpoints used for 9 antifungal agents are listed in Supplementary table 2.

\section{Interpretation of MIC Results}

Interpretation of susceptibility was performed by applying the clinical breakpoints (CBPs) defined by the document M60-2ed [13]. In the absence of CBPs, isolates were defined as having a wild-type (WT) or a non-wild-type (NWT) drug susceptibility phenotype (to amphotericin $\mathrm{B}$, posaconazole, itraconazole and 5-flucytosine) according to the epidemiological cutoff values (ECV) defined by the document M59-3ed [14], as shown in Supplementary table 2.

\section{Ethical Statement}

Ethical approval and patient consensus were not considered necessary due to the descriptive nature of the study that implied only the samples obtained during routine laboratory activity.

\section{Results}

Table 1 summarized the antifungal susceptibility profile of 244 C. albicans isolates to 9 antifungals. The MIC geometric means of the antifungals across all isolates were the following (in increasing order): micafungin $(0.048 \mathrm{mg} / \mathrm{L})$, anidulafungin $(0.132 \mathrm{mg} /$ $\mathrm{L})$, caspofungin $(0.19 \mathrm{mg} / \mathrm{L})$, itraconazole $(0.23 \mathrm{mg} /$ $\mathrm{L})$, posaconazole $(0.25 \mathrm{mg} / \mathrm{L})$, voriconazole (0.28 mg/L), 5-flucytosine $(0.44 \mathrm{mg} / \mathrm{L})$, amphotericine $B(0.49 \mathrm{mg} / \mathrm{L})$ and fluconazole $(2.01 \mathrm{mg} / \mathrm{L})$.

Table 2 summarized MIC distribution, resistance rate and NWT rate of 244 C. albicans isolates from VVC to 9 antifungal agents. Of the 244 C. albicans isolates, $86 \%$ (210) were susceptible to the three echinocandins tested, and $6.5 \%$ of the C. albicans isolates were resistant to anidulafungin which was much higher than resistance rates obtained for the two other echinocandins tested $(0.4 \%$ and $0.8 \%$, respectively).

The in vitro activity of triazoles against 244 isolates of $C$. albicans was variable. Fluconazole was active against $59.4 \%(145 / 244)$ of the isolates tested, and $25.8 \%(63 / 244)$ of the C. albicans isolates were resistant to fluconazole. However, 14.8\% (36/244) of the isolates were fluconazole SDD. Voriconazole had reduced activity to approximately more than half of the isolates with a susceptibility rate of $34.8 \%$ (85/ $244)$, whereas $51.6 \%(126 / 244)$ of the isolates exhibited an intermediate susceptibility and $13.5 \%(33 / 244)$ were resistant to voriconazole. Resistance to both fluconazole and voriconazole was found in 23 isolates of C. albicans. However, $90.5 \%$ of the C. albicans isolates showed relatively higher MICs than 
Table 1 Antifungal susceptibility profile of 244 C. albicans isolates from VVC to 9 antifungal agents

\begin{tabular}{|c|c|c|c|c|c|c|c|c|}
\hline Drug & Range & $\mathrm{MIC}_{50} / \mathrm{MIC}_{90}$ & GM & $\mathrm{S}(\%)$ & I $(\%)$ & $\operatorname{SDD}(\%)$ & $\mathrm{R}(\%)$ & NWT (\%) \\
\hline MFG & $0.016-1$ & $0.0313 / 0.25$ & 0.048 & $227 / 93.0 \%$ & $15 / 6.2 \%$ & NA & $2 / 0.8 \%$ & NA \\
\hline ANF & $0.016-4$ & $0.125 / 0.5$ & 0.132 & $210 / 86.1 \%$ & $18 / 7.4 \%$ & NA & $16 / 6.5 \%$ & NA \\
\hline CAS & $0.0313-1$ & $0.25 / 0.25$ & 0.191 & $222 / 91.0 \%$ & $21 / 8.6 \%$ & NA & $1 / 0.4 \%$ & NA \\
\hline FLC & $0.125-64$ & $2 / 16$ & 2.011 & $145 / 59.4 \%$ & NA & $36 / 14.8 \%$ & $63 / 25.8 \%$ & NA \\
\hline VRC & $0.125-4$ & $0.25 / 1$ & 0.278 & $85 / 34.8 \%$ & $126 / 51.6 \%$ & NA & $33 / 13.5 \%$ & NA \\
\hline POS & $0.0313-4$ & $0.25 / 1$ & 0.251 & $\mathrm{NA}^{\mathrm{a}}$ & NA & NA & NA & $221 / 90.5 \%$ \\
\hline ITR & $0.0313-16$ & $0.25 / 1$ & 0.232 & NA & NA & NA & NA & NA \\
\hline $\mathrm{AmB}$ & $0.25-1$ & $0.5 / 0.5$ & 0.485 & NA & NA & NA & NA & $0 / 0 \%$ \\
\hline $5-\mathrm{FC}$ & $0.125-64$ & $0.25 / 2$ & 0.436 & NA & NA & NA & NA & NA \\
\hline
\end{tabular}

a, not applicable; GM, geometric mean values; S\%, susceptible rate; I\%, intermediate susceptible rate; SDD $\%$, susceptible dose dependent rate; $\mathrm{R} \%$, resistant rate; NWT\%, rate of non-wild type

Table 2 MIC distribution, R\%, NWT\% of 244 C. albicans isolates from VVC to 9 antifungal agents

\begin{tabular}{lclrrrrrrrrrrrrr}
\hline Drug & 0.016 & 0.0313 & 0.0625 & 0.125 & 0.25 & \multicolumn{1}{l}{0.5} & \multicolumn{1}{c}{1} & \multicolumn{1}{c}{2} & \multicolumn{1}{l}{4} & \multicolumn{1}{c}{8} & 16 & 32 & $\geq 64$ & R\% & NWT\% \\
\hline MFG & 16.4 & $61.5^{\mathrm{a}}$ & 80.7 & 87.3 & $93.0^{\mathrm{b}}$ & 99.2 & 100.0 & 100.0 & 100.0 & 100.0 & 100.0 & 100.0 & 100.0 & $0.8 \%$ & $38.50 \%$ \\
ANF & 3.3 & 8.2 & 37.3 & 67.6 & 86.1 & 93.4 & 97.1 & 99.2 & 100.0 & 100.0 & 100.0 & 100.0 & 100.0 & $6.5 \%$ & $32.4 \%$ \\
CAS & 0.0 & 2.9 & 10.7 & 34.4 & 91.0 & 99.2 & 100.0 & 100.0 & 100.0 & 100.0 & 100.0 & 100.0 & 100.0 & $0.4 \%$ & NA \\
FLC & 0.0 & 0.0 & 0.0 & 5.7 & 13.9 & 25.4 & 42.6 & 59.4 & 74.2 & 86.1 & 92.2 & 99.6 & 100.0 & $25.8 \%$ & $74.6 \%$ \\
VRC & 0.0 & 0.0 & 0.0 & 34.8 & 66.0 & 86.5 & 98.0 & 99.2 & 100.0 & 100.0 & 100.0 & 100.0 & 100.0 & $13.5 \%$ & $100 \%$ \\
POS & 0.0 & 5.3 & 23.0 & 42.2 & 59.4 & 81.6 & 90.2 & 97.5 & 100.0 & 100.0 & 100.0 & 100.0 & 100.0 & NA & $90.5 \%$ \\
ITR & 0.0 & 14.8 & 24.2 & 39.8 & 60.2 & 84.4 & 93.0 & 95.9 & 98.8 & 99.6 & 100.0 & 100.0 & 100.0 & NA & NA \\
AmB & 0.0 & 0.0 & 0.0 & 0.0 & 11.5 & 93.0 & 100.0 & 100.0 & 100.0 & 100.0 & 100.0 & 100.0 & 100.0 & NA & 0 \\
5-FC & 0.0 & 0.0 & 0.0 & 35.7 & 51.6 & 66.0 & 76.2 & 95.5 & 98.0 & 98.8 & 99.2 & 98.2 & 100.0 & NA & NA \\
\hline
\end{tabular}

a, percentage of isolates with MIC $\leqq \mathrm{ECV} ; \mathrm{b}$, percentage of isolates with MIC $\leqq \leqq$ susceptible CBP; NA, not applicable; R\%, resistant rate; NWT\%, rate of non-wild type

epidemiological cutoff value (ECV $0.06 \mathrm{mg} / \mathrm{L}$ ) to posaconazole. Regarding the susceptibility to itraconazole, $60.2 \%$ of the isolates had MIC values $>0.125 \mathrm{mg} / \mathrm{L}$, and $15.6 \%$ had $\mathrm{MIC}$ values $>0.5 \mathrm{mg} / \mathrm{L}$.

As expected, all C. albicans isolates tested revealed lower MICs than ECV (2 mg/L) to amphotericin B, while $98.0 \%$ of isolates showed MIC lower than $4 \mathrm{mg} /$ L to 5-flucytosine.

The genotypic relationship of $244 C$. albicans isolates from VVC patients was determined based on UPGMA analysis of 3 microsatellite markers CEF3, CAIII, LOC4. Seven clades were recognized (Fig. 1). Microsatellite genotyping of three loci showed considerable diversity among 244 C. albicans isolates, and 129 different allelic combinations were identified among 244 unrelated C. albicans isolates with 108 singleton genotypes (Supplementary table 3). The combined discriminatory power (DP) of the 3-loci (CAIII, CEF3, and LOC4) typing method was 0.96. The most frequent genotype was genotype A (34/244, $13.9 \%)$ followed by genotypes B $(25 / 244,10 \%)$ and C $(18 / 244,7 \%)$. The other remaining genotypes had a frequency lower than 5\% (Supplementary table 1).

\section{Discussion}

This study investigated the antifungal susceptibility profiles and genetic diversity of 244 C. albicans isolates from VVC patients in Suzhou, eastern China. 




Fig. 1 UPGMA clustering dendrogram of the 244 C. albicans isolates from VVC patients based on the combined analysis of CEF3, CAIII and LOC4 microsatellite markers

The majority of these isolates showed good antifungal activity to the three echinocandins. Micafungin $\left(\mathrm{MIC}_{50} / \mathrm{MIC}_{90}: \quad 0.031 / 0.25 \mathrm{mg} / \mathrm{L}\right)$ showed slightly higher potency than caspofungin $\left(\mathrm{MIC}_{50} /\right.$ $\left.\mathrm{MIC}_{90}: 0.25 / 0.25 \mathrm{mg} / \mathrm{L}\right)$ and anidulafungin $\left(\mathrm{MIC}_{50} /\right.$ $\mathrm{MIC}_{90}: 0.125 / 0.5 \mathrm{mg} / \mathrm{L}$ ) which was consistent with previous studies $[5,15]$. Of note, less than $10 \%$ of $C$. albicans isolates exhibited an intermediate susceptibility to the three echinocandins tested, whereas 16 out of the $244(6.5 \%)$ C. albicans isolates showed resistance mainly to anidulafungin (mono-echinocandin resistance). Furthermore, of the 16 isolates of $C$. albicans with high-MIC anidulafungin phenotype (MIC $\geqq 1 \mathrm{mg} / \mathrm{L}$ ), only one isolate had MIC $\geqq 1 \mathrm{mg}$ / L for micafungin, and another isolate had MIC $\geqq 1$ $\mathrm{mg} / \mathrm{L}$ for caspofungin, which is not in agreement with previous reports indicating that $C$. albicans resistant to echinocandins accounted for less than $<1 \%[6,16]$ and that the mono-echinocandin resistance phenotype was rare [17, 18]. However, our previous study reported one isolate with $\mathrm{MIC} 1 \mathrm{mg} / \mathrm{L}$ for anidulafungin among 207 C. albicans isolates from VVC patients in western China [5]. Pfaller et al. [19] presented similar results on anidulafungin against $C$. albicans causing invasive infections. Lindberg et al. [20] determined the in vitro susceptibility of Candida isolates from the blood samples of patients with candidemia at a Swedish hospital and found that $17 \%$ of $C$. albicans isolates were not susceptible to anidulafungin by applying the EUCAST CBPs. However, when the CLSI CBPs were applied, all the isolates exhibited susceptibility to anidulafungin. Thus, isolates with this high anidulafungin MIC warrant further study.

All triazoles tested had reduced activity against $C$. albicans isolates from VVC patients. Voriconazole had the lowest susceptibility rate of $34.8 \%(85 / 244)$, followed by fluconazole 59.4\% (145/244), respectively. Of note, about half of $C$. albicans isolates $(51.6 \%)$ tested were classified as exhibiting an intermediate susceptibility to voriconazole, whereas $25.8 \%$ and $13.5 \%$ of the isolates were resistant to fluconazole and voriconazole, respectively. Compared to our previous study in western China [5], fluconazole 
resistance of $C$. albicans isolates from VVC significantly increased in eastern China (8.2\% resistance rate in western China versus $25.8 \%$ resistance rate in Suzhou, eastern China). Our results were also compatible with most Chinese reports indicating that approximately half of $C$. albicans isolates causing VVC were susceptible to fluconazole [21-23]. Notably, percentages of isolates with resistance and I/SDD to fluconazole and voriconazole in Suzhou were much higher than those in previous reports from Boikov et al. [15], Ying et al. [21] and Shi et al. [23].

The MIC values for posaconazole in present study were high with $90.5 \%$ NWT isolates which was higher than $60 \%$ NWT isolates found in our previous study in west area when the CLSI ECV $(0.06 \mathrm{mg} / \mathrm{L})$ was applied. Our findings are conflicting with those from North America [24] and Kuwait [25] which showed good activity of posaconazole against $C$. albicans isolates from VVC ( $\mathrm{MIC}_{90}: 0.03 \mathrm{mg} / \mathrm{L}$ and $0.064 \mathrm{mg} /$ $\mathrm{L}$, respectively). However, $37.6 \%$ NWT isolates to posaconazole were reported from invasive candidiasis [26]. Although there was no interpretive breakpoint for itraconazole to $C$. albicans based on the newly described CLSI breakpoints [13, 14], our findings are unusual since $60 \%$ isolates had MIC value $>0.125$ $\mathrm{mg} / \mathrm{L}$, and $15.6 \%(38 / 244)$ isolates had MIC value $\geqq 1 \mathrm{mg} / \mathrm{L}$ (Table 2). Overall, resistance to triazoles among C. albicans isolates was found to be increasing over time, and this could be associated with frequent usage of these azoles in clinical settings in Suzhou area [27], Therefore, a continued surveillance on the antifungal susceptibility among $C$. albicans is necessary to guide treatment of VVC.

Microsatellite genotyping of three loci showed considerably high diversity, and 129 different allelic combinations were identified among 244 isolates of $C$. albicans recovered from patients with VVC. Seven clades were recognizable based on a categorical analysis of CEF3, CAIII, LOC4 microsatellite markers in combination with UPGMA clustering (Fig. 1). We observed that the genotypes A-C (77/244) accounted for $31.5 \%$ of the isolates. Moreover, a total of 108 isolates were shown to represent unique molecular types which account for $44 \%$ of the isolates tested, indicating high genetic diversity within the isolates in this study (Supplementary table 1). Our results confirmed the high genetic diversity among C. albicans isolates reported in previous similar studies $[10,11]$. Sharifynia et al. [11] analyzed the different allelic combinations of 3 microsatellite loci (CAIII, CEF3, and LOC) in 105 independent $C$. albicans strains isolated from patients in Iran, and identified 93 unique microsatellite genotypes that clustered into six clades. Garcia-Hermoso et al. [28] identified 38 different genotypes among $50 \mathrm{C}$. albicans strains in a surgical intensive care unit using the microsatellite analysis of EF3, CDC3 and HIS3 loci. The relatively high genetic variability among $C$. albicans samples may be related to high dynamism of the $C$. albicans genome with recombination, rearrangement and rapid adaptation to host and drug resistance [29].

\section{Conclusions}

Drug resistances of $C$. albicans present significant challenges to implement appropriate therapies and treatment for VVC. Therefore, antifungal susceptibility testing of Candida isolates plays a crucial role in the management of Candida infections. The microsatellite data of $C$. albicans confirmed that this medically important yeast has maintained high levels of genetic variability in Eastern China.

Acknowledgements N.H., Y.L. and H.C. contributed equally to this work and should share the first co-authorship.

Author Contributions Conceptualization, N.H., \& SW.D.; Methodology, Y.L., L.Y, H.Z., R.F., DY.H., G.W. \& XF. C.; data analysis, N.H. \& H.C.; writing-original draft preparation, SW.D. \&N.H.; writing-review and editing, SW.D. \& H.C.; visualization, D.H. \& H.C.; supervision, WQ.L. \& SW.D. All authors have read and agreed to the published version of the manuscript.

Funding This study were funded by from Suzhou Health and Family Planning Commission (LCZX201728), Suzhou New District (2017Z008) to Shuwen Deng; partly by two projects of National Natural Science Foundation of China (82102419 and 81720108026) and Innovation Team Foundation of Jiangsu Province (grant number CXTDA2017038).

Data Availability Sequence data from this study are available in Genbank database under the accession number MZ172462MZ172702 and MZ226435-MZ226437.

\section{Declarations}

Conflict of interest The authors declare no conflict of interest. The funders had no role in the design of the study; in the collection, analyses, or interpretation of data; in the writing of the manuscript, or in the decision to publish the results. 
Open Access This article is licensed under a Creative Commons Attribution 4.0 International License, which permits use, sharing, adaptation, distribution and reproduction in any medium or format, as long as you give appropriate credit to the original author(s) and the source, provide a link to the Creative Commons licence, and indicate if changes were made. The images or other third party material in this article are included in the article's Creative Commons licence, unless indicated otherwise in a credit line to the material. If material is not included in the article's Creative Commons licence and your intended use is not permitted by statutory regulation or exceeds the permitted use, you will need to obtain permission directly from the copyright holder. To view a copy of this licence, visit http://creativecommons.org/licenses/by/4.0/.

\section{References}

1. Mendling W, Brasch J, Cornely OA, Effendy I, Friese K, Ginter-Hanselmayer G, Hof H, Mayser P, Mylonas I, Ruhnke M, Schaller M, Weissenbacher ER. Guideline: Vulvovaginal Candidosis (Awmf 015/072), S2k (Excluding Chronic Mucocutaneous Candidosis). Mycoses. 2015;58(Suppl 1):1-15.

2. Achkar JM, Fries BC. Candida infections of the genitourinary tract. Clin Microbiol Rev. 2010;23(2):253-73.

3. Nagashima M, Yamagishi Y, Mikamo H. Antifungal susceptibilities of Candida species isolated from the patients with vaginal candidiasis. $J$ Infect Chemother. 2016;22(2):124-6.

4. Richter SS, Galask RP, Messer SA, Hollis RJ, Diekema DJ, Pfaller MA. Antifungal susceptibilities of candida species causing vulvovaginitis and epidemiology of recurrent cases. J Clin Microbiol. 2005;43(5):2155-62.

5. Yan L, Wang XD, Seyedmousavi S, Yuan JN, Abulize P, Pan WH, Yu N, Yang YL, Hu HQ, Liao WQ, Deng SW. Antifungal susceptibility profile of Candida Albicans isolated from Vulvovaginal candidiasis in Xinjiang Province of China. Mycopathologia. 2019;184(3):413-22.

6. Arendrup MC, Dzajic E, Jensen RH, Johansen HK, Kjaeldgaard P, Knudsen JD, Kristensen L, Leitz C, Lemming LE, Nielsen L, Olesen B, Rosenvinge FS, Røder BL, Schønheyder HC. Epidemiological changes with potential implication for antifungal prescription recommendations for fungaemia: data from a Nationwide Fungaemia Surveillance Programme. Clin Microbiol Infect. 2013;19(8):E343-53.

7. Adjapong G, Hale M, Garrill A. Population structure of Candida Albicans from three teaching hospitals in Ghana. Med Mycol. 2016;54(2):197-206.

8. Ge SH, Wan Z, Li J, Xu J, Li RY, Bai FY. Correlation between azole susceptibilities, genotypes, and Erg11 mutations in Candida Albicans isolates associated with vulvovaginal candidiasis in China. Antimicrob Agents Chemother. 2010;54(8):3126-31.

9. Sampaio P, Gusmão L, Correia A, Alves C, Rodrigues AG, Pina-Vaz C, Amorim A, Pais C. New microsatellite multiplex Pcr for Candida Albicans strain typing reveals microevolutionary changes. J Clin Microbiol. 2005;43(8):3869-76.

10. Güzel AB, Döğen A, Aydın M, Serin A, Serin MS, Kalkancı A, Ilkit M. Genotyping reveals no link between Candida Albicans genotype and vaginitis severity in Turkish Women. Mycopathologia. 2013;175(3-4):287-94.

11. Sharifynia S, Rezaie S, Mohamadnia A, Mortezaee V, Hadian A, Seyedmousavi S. Genetic diversity and antifungal susceptibility of Candida Albicans Isolated from Iranian Patients. Med Mycol. 2019;57(1):127-31.

12. Barbara D. Alexander, MD.MHS. Reference Method for Broth Dilution Antifungal Susceptibility Testing of Yeasts, 4th Edition. Fourth ed. Clinical and Laboratory Standards Iinstitute, 2017.

13. Clinical and Laboratory Standards Institute. Reference Method for Broth Dilution Antifungal Susceptibility Testing of Yeasts: Performance Standards for Antifungal Susceptibility Testing of Yeasts, M60 2nd Edition, 2020.

14. Clinical and Laboratory Standards Institute. Epidemiological Cutoff Values for Antifungal Susceptibility Testing, M59 3nd Edition, 2020.

15. Boikov DA, Locke JB, James KD, Bartizal K, Sobel JD. In Vitro Activity of the Novel Echinocandin Cd101 at Ph 7 and 4 against Candida Spp. Isolates from Patients with Vulvovaginal Candidiasis. J Antimicrob Chemother. 2017;72(5):1355-8.

16. de Aquino Lemos J, Costa CR, de Araújo CR, Souza LK, Silva Mdo R. Susceptibility testing of Candida Albicans isolated from oropharyngeal mucosa of $\mathrm{Hiv}(+)$ patients to fluconazole, amphotericin B and Caspofungin killing kinetics of Caspofungin and Amphotericin B against Fluconazole resistant and susceptible isolates. Braz J Microbiol. 2009;40(1):163-9.

17. Arendrup MC, Perlin DS. Echinocandin resistance: an emerging clinical problem? Curr Opin Infect Dis. 2014;27(6):484-92.

18. Siopi M, Tarpatzi A, Kalogeropoulou E, Damianidou S, Vasilakopoulou A, Vourli S, Pournaras S, Meletiadis J. Epidemiological trends of Fungemia in Greece with a focus on Candidemia during the recent financial crisis: a 10-year survey in a tertiary care academic hospital and review of literature. Antimicrob Agents Chemother. 2020;64(3):e01516-19.

19. Pfaller MA, Boyken L, Hollis RJ, Kroeger J, Messer SA, Tendolkar S, Diekema DJ. In vitro susceptibility of invasive isolates of Candida Spp. to anidulafungin, caspofungin, and micafungin: six years of global surveillance. J Clin Microbiol. 2008;46(1):150-6.

20. Lindberg E, Hammarström H, Ataollahy N, Kondori N. Species distribution and antifungal drug susceptibilities of yeasts isolated from the blood samples of patients with Candidemia. Sci Rep. 2019;9(1):3838.

21. Ying C, Zhang H, Tang Z, Chen H, Gao J, Yue C. Antifungal susceptibility and molecular typing of 115 Candida Albicans isolates obtained from vulvovaginal candidiasis patients in 3 shanghai maternity hospitals. Med Mycol. 2016;54(4):394-9.

22. Liu XP, Fan SR, Peng YT, Zhang HP. Species distribution and susceptibility of candida isolates from patient with vulvovaginal candidiasis in Southern China from 2003 to 2012. J Mycol Med. 2014;24(2):106-11. 
23. Shi XY, Yang YP, Zhang Y, Li W, Wang JD, Huang WM, Fan YM. Molecular identification and antifungal susceptibility of 186 Candida isolates from vulvovaginal candidiasis in Southern China. J Med Microbiol. 2015;64(Pt 4):390-3.

24. Danby CS, Boikov D, Rautemaa-Richardson R, Sobel JD. Effect of $\mathrm{Ph}$ on in vitro susceptibility of Candida Glabrata and Candida Albicans to 11 antifungal agents and implications for clinical use. Antimicrob Agents Chemother. 2012;56(3):1403-6.

25. Alfouzan W, Dhar R, Ashkanani H, Gupta M, Rachel C, Khan ZU. Species spectrum and antifungal susceptibility profile of vaginal isolates of Candida in Kuwait. J Mycol Med. 2015;25(1):23-8.

26. Noni M, Stathi A, Vaki I, Velegraki A, Zachariadou L, Michos A. Changing epidemiology of invasive candidiasis in children during a 10-year period. J Fungi Basel. 2019;5(1):19.
27. Lin XY, Wang SL, Duan XL, Lan CG, Chen XJ, Xue J, Yang Y. Review of clinical experience in itraconazole therapy for 10 years in China. J Clin Dermatol. 2003;32(7):429-30.

28. Garcia-Hermoso D, Cabaret O, Lecellier G, Desnos-Ollivier M, Hoinard D, Raoux D, Costa JM, Dromer F, Bretagne S. Comparison of microsatellite length polymorphism and multilocus sequence typing for DNA-based typing of Candida Albicans. J Clin Microbiol. 2007;45(12):3958-63.

29. Ene IV, Bennett RJ, Anderson MZ. Mechanisms of genome evolution in Candida Albicans. Curr Opin Microbiol. 2019;52:47-54.

Publisher's Note Springer Nature remains neutral with regard to jurisdictional claims in published maps and institutional affiliations. 